\title{
Focused study of thermonuclear bursts on neutron stars
}

\author{
Chenevez, Jérôme
}

Published in:

SIMBOL-X: Focusing on the hard x-ray Universe

Publication date:

2009

Document Version

Publisher's PDF, also known as Version of record

Link back to DTU Orbit

Citation (APA):

Chenevez, J. (2009). Focused study of thermonuclear bursts on neutron stars. In SIMBOL-X: Focusing on the hard $x$-ray Universe: Proceedings of the 2nd International Simbol-X Symposium (Vol. 1126, pp. 328-330). American Institute of Physics.

\section{General rights}

Copyright and moral rights for the publications made accessible in the public portal are retained by the authors and/or other copyright owners and it is a condition of accessing publications that users recognise and abide by the legal requirements associated with these rights.

- Users may download and print one copy of any publication from the public portal for the purpose of private study or research.

- You may not further distribute the material or use it for any profit-making activity or commercial gain

- You may freely distribute the URL identifying the publication in the public portal

If you believe that this document breaches copyright please contact us providing details, and we will remove access to the work immediately and investigate your claim. 


\title{
Focused study of thermonuclear bursts on neutron stars
}

\author{
Jérôme Chenevez \\ National Space Institute, Technical University of Denmark
}

\begin{abstract}
X-ray bursters form a class of Low Mass X-Ray Binaries where accreted material from a donor star undergoes rapid thermonuclear burning in the surface layers of a neutron star. The flux released can temporarily exceed the Eddington limit and drive the photosphere to large radii. Such photospheric radius expansion bursts likely eject nuclear burning ashes into the interstellar medium, and may make possible the detection of photoionization edges. Indeed, theoretical models predict that absorption edges from ${ }^{58} \mathrm{Fe}$ at $9.2 \mathrm{keV},{ }^{60} \mathrm{Zn}$ and ${ }^{62} \mathrm{Zn}$ at $12.2 \mathrm{keV}$ should be detectable by the future missions Simbol-X and NuSTAR. A positive detection would thus probe the nuclear burning as well as the gravitational redshift from the neutron star. Moreover, likely observations of atomic X-ray spectral components reflected from the inner accretion disk have been reported. The high spectral resolution capabilities of the focusing X-ray telescopes may therefore make possible to differentiate between the potential interpretations of the X-ray bursts spectral features.
\end{abstract}

Keywords: X-rays: bursts, stars: neutron

PACS: $26.30 . \mathrm{Ca}$

\section{INTRODUCTION}

The Simbol-X mission, as well as the NuSTAR mission to be launched in 2011, will provide an unprecedented focusing view of the Universe in hard X-rays. In the frame of the NuSTAR Galactic Program a few of the known low-mass X-ray binaries (LMXRBs) will be observed that may exhibit thermonuclear burst events. The National Space Institute of Denmark, which is producing the optics for NuSTAR, has proposed to exploit the improved sensitivity of this instrumentation to study the physics of such events up to hard X-rays.

X-ray bursters form a class of LMXRBs where accreted material, essentially composed of hydrogen and/or helium, from a donor star undergoes rapid thermonuclear burning in the surface layers of a neutron star. Such type I X-ray bursts, characterized by a black-body emission with a temperature, $\mathrm{kT}$, between 1 and $3 \mathrm{keV}$, display generally a fast rise time followed by an exponential decay phase, which duration ranges typically between a few seconds and a few minutes. They have a recurrence time of hours to days, but the detailed relationship between accretion regime, burst types, and recurrence times is still an issue for most of X-ray burst systems. It is a function of accretion rate and composition, depending on the nature of the donor star (its age, size, and composition) and the geometry of the binary system, and of the sedimentation and nuclear ignition mechanisms on the surface of the neutron star. An improved investigation of these various processes will further our knowledge about the composition of the companion stars and therefore the evolution of LMXRBs, in addition to a better understanding of the geometry of the accretion flow onto the neutron stars, the nuclear burning processes, 


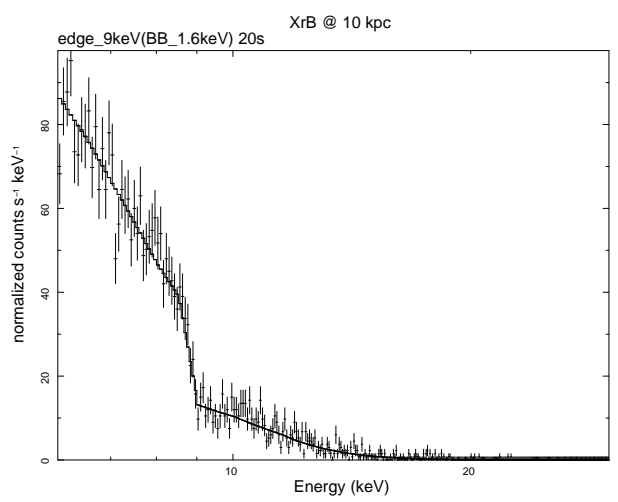

FIGURE 1. Simulation of a $1.6 \mathrm{keV}$ black-body spectrum with an absorption edge at $9 \mathrm{keV}$ of a typical $\mathrm{X}$-ray burst emitted at a distance of $10 \mathrm{kpc}$. The count rate is $212 \mathrm{cts} / \mathrm{s}$.

as well as their interior thermal structure, magnetic field, and spin. The Simbol-X instrumentation should as well be considered to further perform focused observations of $\mathrm{X}$-rays bursts allowing advanced research on their thermonuclear physic processes.

\section{FOCUSING ON NUCLEAR PHYSICS}

The instabilities of nuclear burning in relation with the X-ray bursts have received much theoretical attention [see, e.g. 7, for a review], making clear that much of the accumulated hydrogen and helium is burned to heavy elements. The flux released can temporarily exceed the Eddington limit and drive the photosphere to large radii. Such photospheric radius expansion (PRE) bursts likely eject nuclear burning ashes into the interstellar medium, and may make possible the detection of photoionization edges. Indeed, Weinberg et al. [9] predict that during PRE bursts of He-rich accretors at low accretion rates like $4 \mathrm{U} 1820-30$ [3], absorption edges from ${ }^{58} \mathrm{Fe}$ at $9.2 \mathrm{keV},{ }^{60} \mathrm{Zn}$ and ${ }^{62} \mathrm{Zn}$ at $12.2 \mathrm{keV}$ should be detectable by NuSTAR and Simbol-X.

Fig. 1 shows a simulation of an X-ray burst spectrum with such an edge at $9 \mathrm{keV}$. A positive detection of absorption edges would thus imply that heavy hydrogen-like ions are ejected from the neutron star and would probe the nuclear burning [9]. Moreover, likely observations of atomic X-ray spectral components reflected from the inner accretion disk have been reported [e.g. 8, 5]. The high spectral resolution capabilities of the coming focusing missions may therefore make possible to differentiate between the potential interpretations of the X-ray bursts spectral features.

In addition to the few bursting sources located close to the Galactic Center which bursting activity is not foreseeable, different kind of systems should be included in the observation program, in order to interpret the diversity of the X-ray burst observations. As mentioned above, 4U 1820-30 is likely the best candidate to observe spectral features from He-rich systems. The so-called "clocked burster" GS 1826-238, because its recurrence time is quasi-periodic between $3.5 \mathrm{~h}$ and $5.74 \mathrm{~h}$ [4], is the more obvious candidate to observe X-ray bursts with a focusing telescope. 


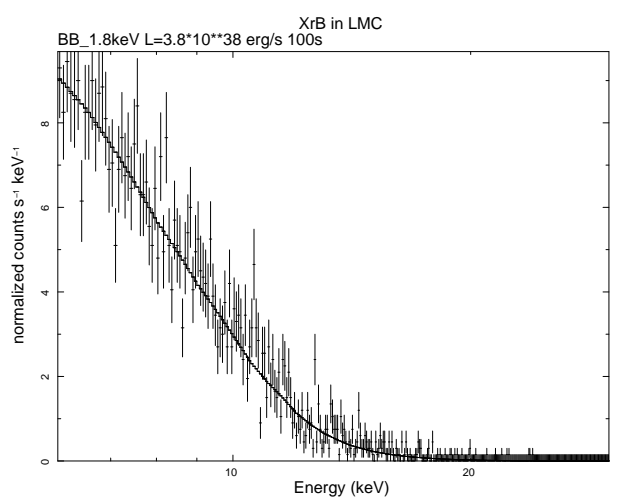

FIGURE 2. Simulation of a $1.8 \mathrm{keV}$ black-body spectrum of a 100s long PRE X-ray burst in LMC. The count rate is $31 \mathrm{cts} / \mathrm{s}$.

Thanks to the high sensitivity of NuSTAR and Simbol-X, events from so-called burstonly sources [e.g. 1, 2], or weak bursts from yet unknown bursting systems may be revealed. Furthermore, Simbol-X as well as NuSTAR, could provide the occasion to explore the Magellanic Clouds for bursting activity outside our own galaxy [see, e.g. 6, who report the observation with XMM of bursts from M31]. As an example, Fig. 2 shows a simulated spectrum of a burst from LMC.

In summary, our main objective is to confront the X-ray burst observational properties with the current theory in order to:

1. resolve the complexity of the relationship between nuclear burning and accretion process regimes,

2. study the effects caused by the burst on the accretion disk around the neutron star,

3 . better understand the nuclear processes involved during X-ray bursts.

\section{ACKNOWLEDGMENTS}

JC acknowledges fruitful discussions with S. Brandt, E. Kuulkers, and H. Schatz. This work was supported by ESA-PRODEX contract N: 90057.

\section{REFERENCES}

1. Cocchi, M., Bazzano, A., Natalucci, L. et al. 2001, A\&A, 378, L37

2. Cornelisse R., et al., A\&A 392, 931, 2002

3. Cumming A., ApJ 595, 1077, 2003

4. Galloway D., et al., ApJ 601, 466, 2004

5. Kuulkers E., et al., A\&A 399, 663, 2003

6. Pietsch W. and Haberl. F, A\&A 430, L45, 2005

7. Strohmayer T. and Bildsten L., in Compact Stellar X-ray sources, Eds. Lewin and van der Klis, 2006 (astro-ph/0301544)

8. Strohmayer T. and Brown E., ApJ 566, 1045, 2002

9. Weinberg N., Bildsten L., and Schatz H., ApJ 639, 1018, 2006 